\title{
Orbital Apex Syndrome due to Orbital Mucormycosis after Teeth Infection: A Successful Case Report
}

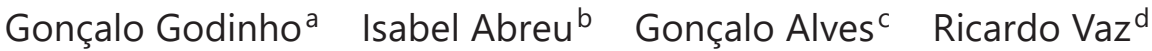 \\ Vitor Leal $^{a} \quad$ Ana Cláudia Carvalho $^{b}$ António Sarmento ${ }^{b}$ \\ Fernando Falcão-Reis ${ }^{a}$ \\ aOphthalmology Department, Centro Hospitalar e Universitário São João, Porto, Portugal; \\ bInfectious Diseases Department, Centro Hospitalar e Universitário São João, Porto, \\ Portugal; ' Neuroradiology Department, Centro Hospitalar e Universitário São João, Porto, \\ Portugal; dORL Department, Centro Hospitalar e Universitário São João, Porto, Portugal
}

\section{Keywords}

Diabetes $\cdot$ Exenteration $\cdot$ Mucorales $\cdot$ Mucormycosis $\cdot$ Teeth

\begin{abstract}
Rhino-orbital-cerebral mucormycosis is a severe and rapidly progressive fungal infection associated with low survival rates. Early diagnosis and proper management are mandatory. We report the case of a 20-year-old female with diabetic ketoacidosis and teeth infection, who presented with sudden orbital apex syndrome. Several surgical procedures were necessary to provide a definite histopathological diagnosis of the fungal infection. Ultimately, given the progressive aggravation of the infection and the ineffectiveness of antifungal and antibiotic agents, an orbital exenteration was performed. A mycobacteriological examination revealed a polymicrobial culture with Mucorales. One year after the initial presentation, the patient is alive and with a good general health condition.
\end{abstract}

\section{Introduction}

Mucormycosis is a rare and severe fungal infection [1] caused by the zygomycete, Mucorales [2]. These ubiquitous saprophytic fungi [3] tend to invade the wall of blood vessels, mainly the arteries, causing intima damage and, consequently, ischemia, and necrosis of the affected tissues [4]. The most common presentation is rhino-orbital-cerebral [5], evolving in 
3 consecutive stages: a generally unnoticed sinus involvement, a symptomatic orbital involvement, and a cerebral involvement [1]. Paranasal sinuses are, generally, the source of the infection although it can also spread through the oral cavity or teeth [6]. The most common risk factor is diabetes mellitus, especially ketoacidosis, although other immunosuppressive conditions and renal failure are known to be predisposing conditions [7, 8].

This is a rapidly progressive and devastating condition, with survival rates of 30-70\% [9]. Consequently, delayed diagnosis and treatment can be disastrous, significantly worsening the prognosis. Hemiparesis, bilateral sinus involvement, leukemia, and renal disease are also associated with lower survival rates [10]. Even in survival situations, significant morbidity and disfigurement are expected. We report the unusual successful case of a nonlethal mucormycosis infection despite the late diagnosis.

\section{Case Report/Case Presentation}

A 20-year-old female with a history of poorly controlled diabetes presented in the emergency department with sudden headache and a painful right eye (OD), associated with hypoesthesia of the upper third of the right hemiface and vomiting. The patient reported having been on antibiotic treatment for the previous 2 weeks (first with amoxicillin/clavulanic acid and then with clarithromycin) because of an active dental infection. The physical examination revealed mild proptosis, right palpebral ptosis, fixed right mydriasis, complete ophthalmoplegia, hypoesthesia of V1 and V2 right territory, and loss of right nasolabial fold (shown in Fig. 1). Visual acuity assessment revealed a highly asymmetrical reduction of best-corrected visual acuity on the right (light perception - OD vs. 20/20 - OE). Biomicroscopy findings were unremarkable, apart from the nonreactive dilated pupil. Fundoscopy showed retinal and choroidal ischemia (shown in Fig. 2), confirmed by subsequent fluorescein angiography. The laboratory workout revealed leukocytosis and diabetic ketoacidosis. The orbital and facial CT-scan showed filling of the right sphenoidal, ethmoidal, and maxillary sinus (compatible with pansinusitis) with ipsilateral extraconical extension (shown in Fig. 3). Afterward, right unciformectomy and maxillary antrostomy followed by 3 dental pieces extraction were performed. Collected samples were sent for histopathological examination and culture, revealing the presence of ampicillin-resistant Klebsiella oxytoca. Antibiotic treatment with endovenous ceftriaxone and clindamycin was implemented.

The patient maintained a stable clinical condition, with pain improvement, facial sensitivity recovery, reduction of the inflammatory markers, and evidence of retinal reperfusion.
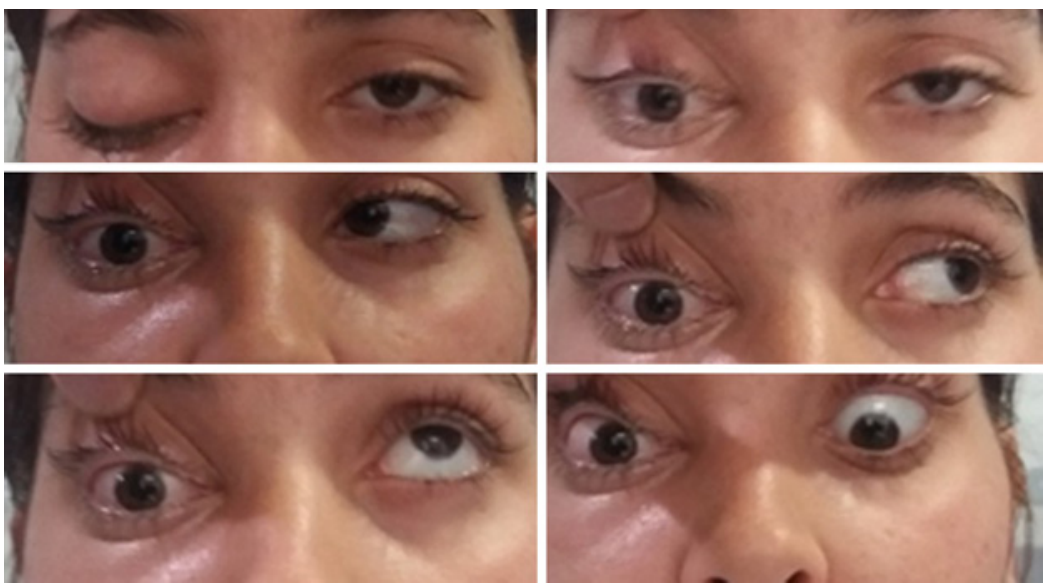

Fig. 1. Mild proptosis, right palpebral ptosis, fixed right mydriasis, and complete ophthalmoplegia.

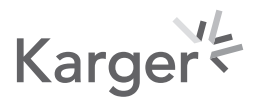


Fig. 2. Retinography showing retinal and choroidal ischemia.

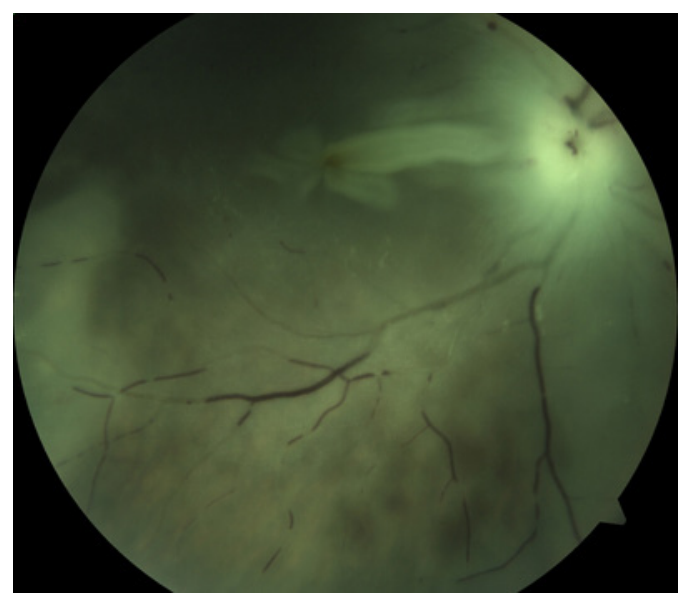

Fig. 3. Face-CT, coronal view: Soft-tissue opacification of the right maxillary sinus extending to the ipsilateral ethmoidal cells. Bone remodeling of the orbit floor, with nodular infiltration of the adjacent extraconic space (white arrow).

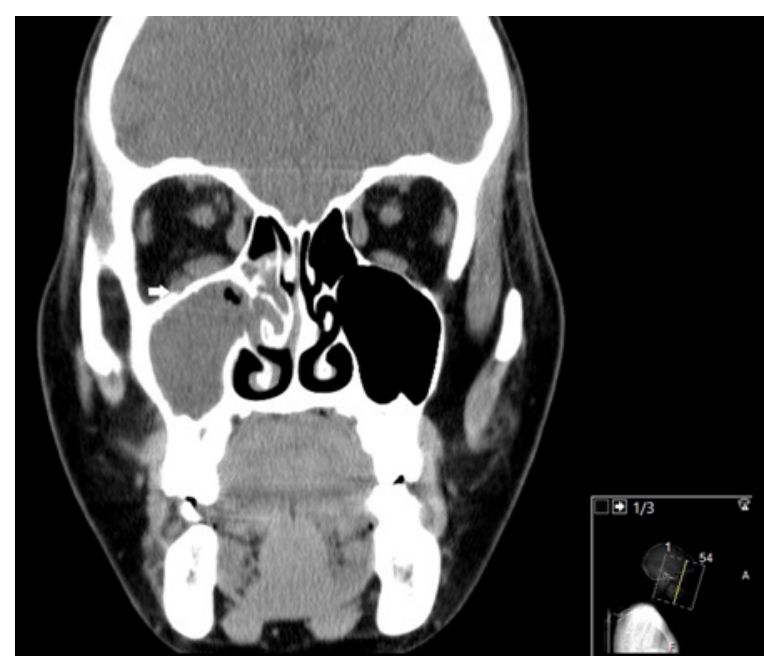

Nevertheless, visual acuity of light perception in OD, ptosis, and ophthalmoplegia remained. Twenty days after antibiotic discontinuation, she developed rhinorrhea and purulent exudation of the previously manipulated dental region. A CT-scan showed a right hemiface abscess extending to the apex of the orbit. Endoscopic ethmoidectomy and orbital exploration with drainage of the abscess were carried out. A new anatomopathological exam was then performed, revealing signs of chronic inflammation. No infectious pathogen was exposed though. The patient began a course of amphotericin B, vancomycin, and meropenem. However, 5 days later, she developed fever and OD pain, and a CT-scan revealed an expansion of the abscess with intra- and extraconical components. Hence, an orbitotomy and endoscopic nasosinusal surgeries with an anatomopathological study were performed, revealing the presence of Candida albicans and Staphylococcus aureus. Even with ampicillin, amphotericin B, and metronidazole, the patient maintained fever. A new orbitotomy with large debridement of orbital tissue was performed after 6 days, revealing the presence of fungal structures.

The patient remained afebrile for 15 days, later developing rhinorrhea, evidencing the progression of the right orbital infection (shown in Fig. 4). It was decided to perform exenteration of the orbit, and the mycobacteriological study revealed Serratia marcescens, Staphylococcus epidermidis, and Mucorales. Accordingly, the patient completed a 4-month cycle of amphotericin B, isavuconazole, teicoplanin, and cefepime. Adjunctive treatment with 
Fig. 4. Brain MRI, axial T1 with gadolinium. Diffuse infectious process of the orbit, infiltrating the fat, muscles, globe coats, lacrimal sac, and duct. Posterior extension to the cavernous sinus (white arrow) and along the dura mater of the ipsilateral anterior temporal lobe (black arrow).

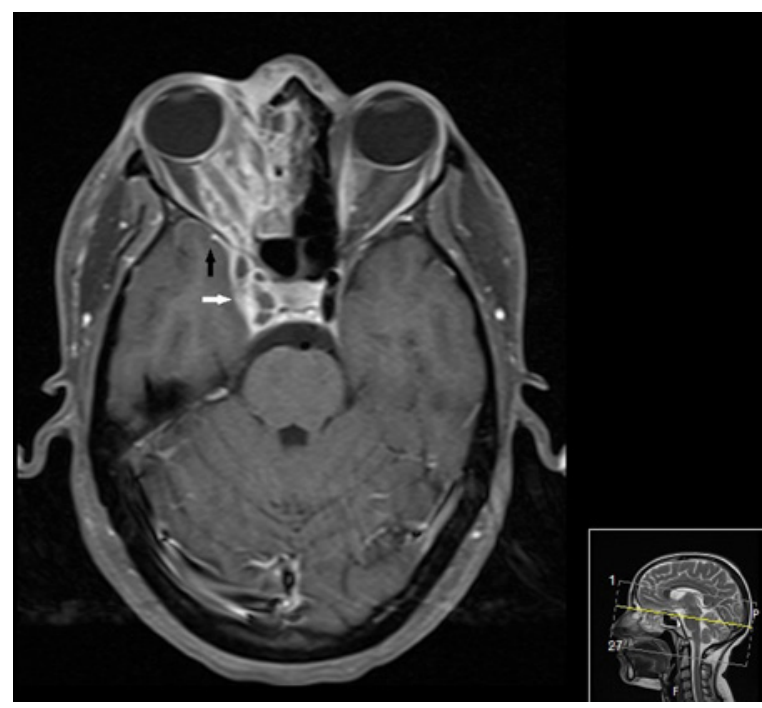

hyperbaric oxygen was also performed. Also, the glycemic status was stable during the followup. The infection was cured, and the patient survived. One year after the surgery, she is in a good general condition.

\section{Discussion/Conclusion}

This case exemplifies a typical infection, occurring in a diabetic patient with poor metabolic control and diabetic ketoacidosis. Acidosis disrupts the iron-binding of transferrin, increasing the amount of unbound iron and promoting fungus growth [1]. Also, the polymorphonuclear dysfunction in diabetic patients, characterized by a delayed margination, encourages fungal proliferation [11]. Additionally, diabetic bronchoalveolar macrophages present a diminished ability to inhibit spore germination [12].

Generally, infection is acquired by inhalation of spores. Here, though, it was presumed to start in the long-standing teeth infection, with subsequent spread into the paranasal sinus and then orbital structures.

Mucormycosis is characterized by vascular tropism because spores and germ tubes adhere to and damage endothelial cells [13]. This phenomenon may lead to ischemic necrosis of host tissues, namely the orbital walls, ocular nerves, and the ophthalmic artery. Ophthalmic signs and symptoms of this disease are variable, with ophthalmoplegia, vision impairment, and proptosis being the most common [14]. Other symptoms include periorbital pain and chemosis [2]. Several other nonophthalmic signs include sinusitis, nasal discharge, facial swelling, cranial nerve VII palsy, and palatal necrosis. In this case, the simultaneous dysfunction of the optic nerve and the cranial nerves III, IV, VII, V1, and V2, along with signs of retinal and choroidal ischemia seen in fundoscopy, were highly suggestive of an orbital apex syndrome with unilateral involvement. This entity is a well-recognized presentation of mucormycosis, but other etiologies could also be considered. In agreement with the various examinations negative for fungus and positive for bacteria, antibacterial agents were prescribed.

The definitive diagnosis was based on histopathological identification of fungal structures and positive culture for Mucorales. Typically, large nonseptate mycelial filaments branching at a right angle are visible [1]. However, the rates of successful tissue cultures are only 33-50\% [15] probably because of hyphae damaging during manipulation, suboptimal 
standard culture conditions, and prolonged antifungal pretreatment $[16,17]$. This low rate of positive cultures for mucormycosis could lead to misleading conclusions and consequently delay the diagnosis. During the acute phase, several surgeries were necessary to improve the general condition and provide a diagnosis. This situation led to a delay in the diagnosis. Although already reported $[6,18]$, polymicrobial cultures are uncommon. So, despite the variety of pathogens identified in the mycobacteriological study, we considered Mucorales the causal agent in the presenting case. Accordingly, adequate antifungal therapy was provided based on that assumption.

Treatment of Mucormycosis requires a multidisciplinary approach. Major risk factors, such as ketoacidosis or hyperglycemia, must be controlled. Although the delivery of the drugs to the necrotic tissue may not be an easy task, medical treatment with systemic antifungal agents is essential, with reported survival rates of $72 \%$ [1]. However, due to vascular tropism, it may be difficult to deliver the drugs to the necrotic tissue. Liposomal amphotericin B is the first choice of treatment, but it requires careful monitoring due to its renal toxicity. Posaconazole is another effective drug [2].

Early and aggressive debridement of necrotic or infected tissue is of critical importance. Surgical treatment alone has a mortality rate of $11 \%$, with this value rising to $60 \%$ in patients not submitted to surgery [19]. In this case, the multiple surgical interventions to remove the infected tissues could have contributed to the survival of the patient, even though no fungal agent was identified. A more invasive strategy, such as surgical exenteration could also be necessary to diminish fungal load and avoid direct cerebral extension [2]. Given the lack of response to previous treatment modalities, it was the way to stop the progression of the infection. Other treatment options, such as the hyperbaric chamber, are advocated in an attempt to provide oxygen flow to ischemic tissues, therefore, improving neutrophil activity and promoting wound healing [20].

Fungal infections are rare entities, generally associated with poor prognosis. A multidisciplinary approach between the departments of ophthalmology, otorhinolaryngology, infectious disease, endocrinology, neurology, neuroradiology, and pathology was critical to obtain the best possible outcome. Multiple factors are associated with a better prognosis, namely, early diagnosis, antifungal therapy, and aggressive debridement of necrotic and infected tissue. In this manuscript, we report such a rare case of an extensive mucormycosis infection witch ultimately had a good outcome despite the delay in the diagnosis.

\section{Statement of Ethics}

This research was conducted ethically in accordance with the World Medical Association Declaration of Helsinki. Written informed consent for publication of the patient's clinical details was obtained.

\section{Conflict of Interest Statement}

The authors have no conflicts of interest to declare.

\section{Funding Sources}

No funding sources to declare.

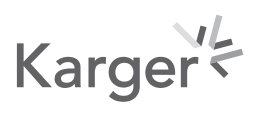


Godinho et al.: Orbital Mucormycosis after Teeth Infection

\section{Author Contributions}

All the authors whose names appear on the submission (1) made substantial contributions to the conception or design of the work; or the acquisition, analysis, or interpretation of data; or the creation of new software used in the work; (2) drafted the work or revised it critically for important intellectual content; and (3) approved the version to be published.

\section{References}

1 Jiang N, Zhao G, Yang S, Lin J, Hu L, Che C, et al. A retrospective analysis of eleven cases of invasive rhino-orbitocerebral mucormycosis presented with orbital apex syndrome initially. BMC Ophthalmol. 2016;16(1):10.

2 Karadeniz Uğurlu S, Selim S, Kopar A, Songu M. Rhino-orbital mucormycosis: clinical findings and treatment outcomes of four cases. Turk J Ophthalmol. 2015;45(4):169-74.

3 Fahrenkopf MP, Nelson JJ, Eichhorn M, Conway J, Hassan A. Rhino-orbital-cerebral mucormycosis and orbital exenteration. Eplasty. 2016;16:ic42.

4 Joos ZP, Patel BC. Intraorbital irrigation of amphotericin B in the treatment of rhino-orbital mucormycosis. Ophthal Plast Reconstr Surg. 2017;33(1):e13-6.

5 Hosseini SM, Borghei P. Rhinocerebral mucormycosis: pathways of spread. Eur Arch Otorhinolaryngol. 2005; 262(11):932-8.

6 Prabhu S, Alqahtani M, Al Shehabi M. A fatal case of rhinocerebral mucormycosis of the jaw after dental extractions and review of literature. J Infect Public Health. 2018;11(3):301-3.

7 Gumral R, Yildizoglu U, Saracli MA, Kaptan K, Tosun F, Yildiran ST. A case of rhinoorbital mucormycosis in a leukemic patient with a literature review from Turkey. Mycopathologia. 2011;172(5):397-405.

8 Ibrahim AS, Spellberg B, Edwards J. Iron acquisition: a novel perspective on mucormycosis pathogenesis and treatment. Curr Opin Infect Dis. 2008;21(6):620-5.

9 Bakshi SS. Rhino-orbital mucormycosis. Bull Emerg Trauma. 2019;7(1):88-9.

10 Yohai RA, Bullock JD, Aziz AA, Markert RJ. Survival factors in rhino-orbital-cerebral mucormycosis. Surv Ophthalmol. 1994;39(1):3-22.

11 Sheldon $\mathrm{WH}$, Bauer $\mathrm{H}$. The development of the acute inflammatory response to experimental cutaneous mucormycosis in normal and diabetic rabbits. J Exp Med. 1959;110:845-52.

12 Waldorf AR, Ruderman N, Diamond RD. Specific susceptibility to mucormycosis in murine diabetes and bronchoalveolar macrophage defense against Rhizopus. J Clin Invest. 1984;74(1):150-60.

13 Ibrahim AS, Spellberg B, Avanessian V, Fu Y, Edwards JE. Rhizopus oryzae adheres to, is phagocytosed by, and damages endothelial cells in vitro. Infect Immun. 2005;73(2):778-83.

14 Prakash H, Chakrabarti A. Global epidemiology of mucormycosis. J Fungi. 2019;5(1):26.

15 Badiee P, Arastefar A, Jafarian H. Comparison of histopathological analysis, culture and polymerase chain reaction assays to detect mucormycosis in biopsy and blood specimens. Iran J Microbiol. 2013;5(4):406-10.

16 Spellberg B, Edwards J Jr, Ibrahim A. Novel perspectives on mucormycosis: pathophysiology, presentation, and management. Clin Microbiol Rev. 2005;18(3):556-69.

17 Tarrand JJ, Han XY, Kontoyiannis DP, May GS. Aspergillus hyphae in infected tissue: evidence of physiologic adaptation and effect on culture recovery. J Clin Microbiol. 2005;43(1):382-6.

18 Vijayabala GS, Annigeri RG, Sudarshan R. Mucormycosis in a diabetic ketoacidosis patient. Asian Pac J Trop Biomed. 2013;3(10):830-3.

19 Tedder M, Spratt JA, Anstadt MP, Hegde SS, Tedder SD, Lowe JE. Pulmonary mucormycosis: results of medical and surgical therapy. Ann Thorac Surg. 1994;57(4):1044-50.

20 Mohamed MS, Abdel-Motaleb HY, Mobarak FA. Management of rhino-orbital mucormycosis. Saudi Med J. 2015;36(7):865-8. 